\title{
URANS and ACM for determining the aerodynamic performance of vertical-axis wind turbine
}

\author{
Krzysztof Rogowski ${ }^{1, *}$, and Martin O. L. Hansen ${ }^{2}$ \\ ${ }^{1}$ Institute of Aeronautics and Applied Mechanics, Warsaw University of Technology, \\ 00-665 Warsaw, Poland \\ ${ }^{2}$ DTU Wind Energy, Technical University of Denmark, Lyngby, 2800 Kgs., Denmark
}

\begin{abstract}
This paper presents numerical results of a one-bladed vertical axis wind turbine using the $k-\varepsilon$ RNG turbulence model. The rotor geometry was developed based on the research report available on the literature. The obtained results of aerodynamic blade loads and wake profiles downstream behind the rotor are in a good agreement with the experiment. This paper presents also the ability of calculating fluid flow parameters around the operating wind turbine by using the original approach - the actuator cell model (ACM).
\end{abstract}

\section{Introduction}

The depletion of global reserves of fossil fuels has resulted in an increase in ecological awareness of the society and an increase in interest in alternative energy sources. The use of wind energy, sometimes referred to as "indirect solar energy", is a promising technology capable of delivering a portion of energy demand in many regions of the world. In the future, wind turbines should be better adapted to the conditions of developing countries because wind energy is the cheapest source of clean energy offering carbon-free generation at the level of costs currently comparable to gas. Wind turbines very effectively provide centralized electricity because they are concentrated in large clusters (wind farms) thanks to which energy is supplied via local distribution grid to local energy companies. European countries such as Germany, Denmark or Spain are the main wind energy beneficiaries. In 2017 the percentage of electricity produced by wind power was already above $40 \%(44 \%)$ in DK and this number is increasing with new wind farms planned. The UK, which has the largest wind resources in Europe, aspires to bring down the price of wind energy. According to the global wind report published by the Global Wind Energy Council (GWEC), in 2017 about $54 \mathrm{GW}$ of power was installed wind turbines. The report forecasts that this amount may increase to even $800 \mathrm{GW}$ in 2021 [1].

The Darrieus vertical-axis wind turbine uses lift force to drive. Thanks to its high aerodynamic efficiency, this turbine is competitive compared to a classic propeller-type

\footnotetext{
${ }^{*}$ Corresponding author: krogowski@meil.pw.edu.pl
} 
horizontal-axis wind turbine. It has been proven that the Darrieus wind turbine is one of the most effective wind energy conversion systems $[2,3]$.

Numerical models for wind turbines with a vertical axis of rotation have been developed for a long time. Strickland was one of the first who began to develop the vortex model for Darrieus-type rotors [2]. This class of aerodynamic models basing on vorticity equations much better predicted overall Darrieus wind turbine performance than commonly known one-dimension momentum based aerodynamic models [3].

Numerical methods of fluid dynamics have become an important tool in the study of aerodynamic properties of wind turbines [4-7]. However, these methods are limited due to the size of the rotor. They also have limitations due to overestimation of the critical angle of attack. CFD methods require a large computer infrastructure to carry out efficient research on a small three-dimensional rotor. It involves not only high computer costs but also software licences and electricity expenses. Therefore, most studies using CFD published until the end of 2018 only concern the modelling of the aerodynamic performance of rotors of VAWT in 2D. Another possibility would be to use system identification methods, however this approach is not possible due to high cost of the experiments $[8,9]$.

Currently there is a big focus on the offshore wind energy sector and his includes Darrieus wind turbines. One example is the DeepWind project, which involves the use of Darrieus floating rotors to generate electricity $[10,11]$. This entails the need to create new analytical tools for the aerodynamic analysis of VAWTs.

The authors of this article intend to validate the classic CFD approach with available experimental results and then validate the original method using the numerical results and experimental results. This article aims to compare the velocity fields obtained using two numerical approaches: URANS with the ReNormalisation Group $(R N G) k-\varepsilon$ model and the actuator cell model developed by Rogowski $[12,13]$.

\section{Numerical model}

The numerical model of a one-bladed rotor was developed based on the experiment of Strickland et al [2]. The experiment was performed in a water towing tank. The rotating single-bladed rotor was moved by means of a special chain mechanism.
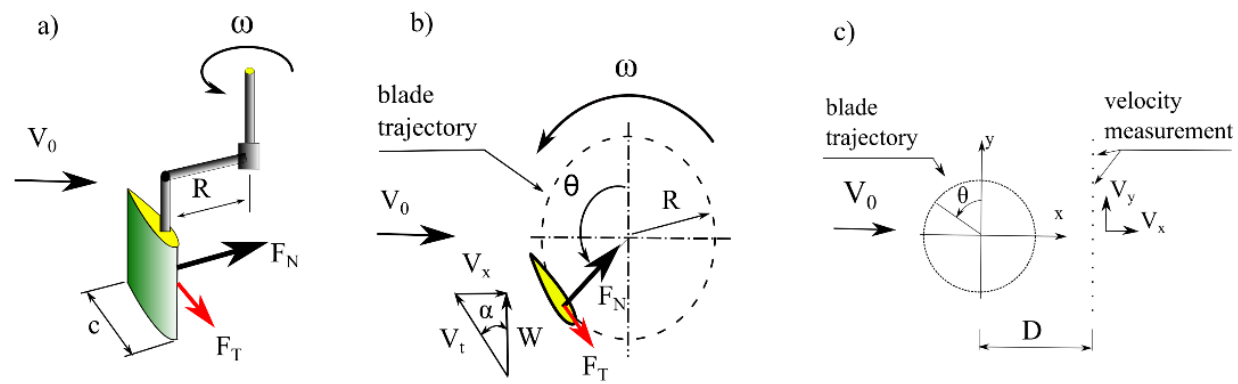

Fig. 1. One-bladed vertical-axis wind turbine (a); velocity vectors and aerodynamic blade loads (b); method of velocity measurement (c).

The rotor configuration consists of a blade constructed using the NACA 0012 profile with the chord length, $c$ of $9.14 \mathrm{~cm}$ and the pitch angle of $0 \mathrm{deg}$. The intention of Strickland et al. [2] was to conduct a two-dimensional experiment, therefore the rotor blades were very long in comparison to the chord length. The rotor diameter $D=2 R$ was $1.22 \mathrm{~m}$. During 
numerical simulations water was used as an operating fluid. The angular velocity of the rotor $\omega$ was $0.75 \mathrm{rad} / \mathrm{s}$. The water velocity at the inlet was chosen to be $9.1 \mathrm{~cm} / \mathrm{s}$. This value corresponds to the tip speed ratio of 5.0. In the experiment the aerodynamic blade loads were measured using strain gage bridges. Moreover, during the experiment, wake velocity profiles were measured downstream behind the rotor. The rotor's silhouette is shown in Fig. 1a. Wake velocity profiles were also computed by CFD using checkpoints behind the wind turbine (Fig. 1c).

\section{Method}

In this work, the unsteady Reynolds-averaged Navier-Stokes (URANS) approach was used to calculate the flow parameters and aerodynamic loads on the blades. In addition, this paper presents the operation of the actuator cell model (ACM) developed by Rogowski [13] for determining flow parameters around the Darrieus-type rotor. Both the URANS and ACM approaches were implemented using the ANSYS Fluent package.

In the URANS approach, the k- $\varepsilon$ RNG turbulence model was used for the calculations. It is two-equation turbulence model that adds two transport equations for the turbulence kinetic energy $k$ and its dissipation $\varepsilon$. Figure 2a presents the concept of this approach. Inside the square computational domain a smaller domain with a circular shape rotates. Data between these domains is exchanged thanks to the interface.

The ACM model differs from the URANS approach in that the rotor blade is not modelled. An additional third very small computing domain is introduced. This additional third domain rotates during simulation around the rotor axis of rotation at the angular velocity equal to the rotor angular velocity. The aerodynamic blade loads are introduced into this third computational domain as momentum sources.

a)

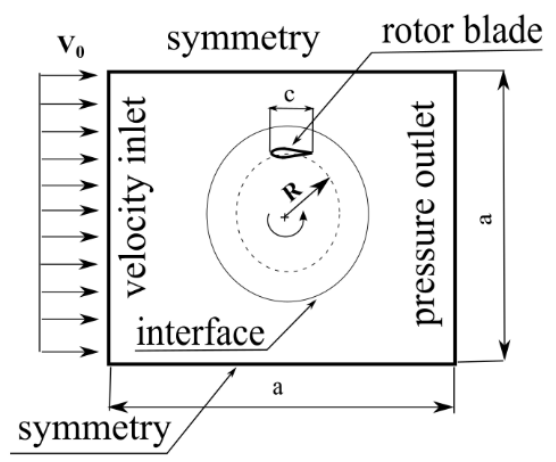

b)

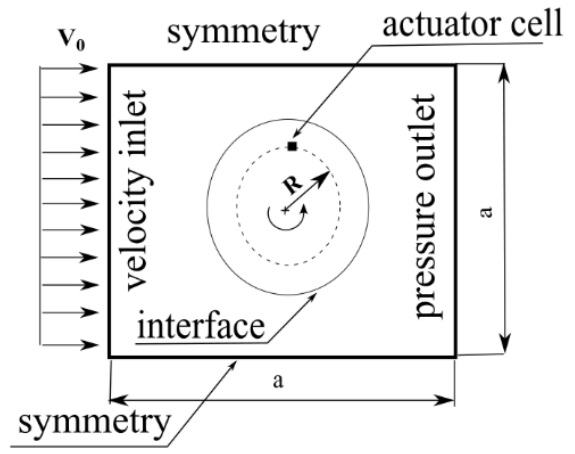

Fig. 2. RANS approach (a); Actuator cell model (b).

Both grids consist of unstructured quadrilateral elements in the whole domain. In the URANS approach the structured mech near the airfoil blade edge is generated. This structured grid provides a better determination of the flow parameters in the boundary layer. However, it gives more elements, which increases the cost of calculations. For all simulations presented here, the wall $\mathrm{y}+$ was lower than 0.8 . The total number of mesh cells for ACM approach is 43,707 elements whereas for URANS approach the number of elements is 131,958 . Transient calculations require the determination of the time step size. In these calculations, the step size corresponds to the increment of azimuthal angle $\Delta \theta=0.1 \mathrm{deg}$. Figure 3a presents the mesh distribution for URANS model whereas the mesh 
for the ACM is given in Fig. 3b. Both grids were examined in terms of element density. More details on these grids can be found in $[12,13]$.

a)

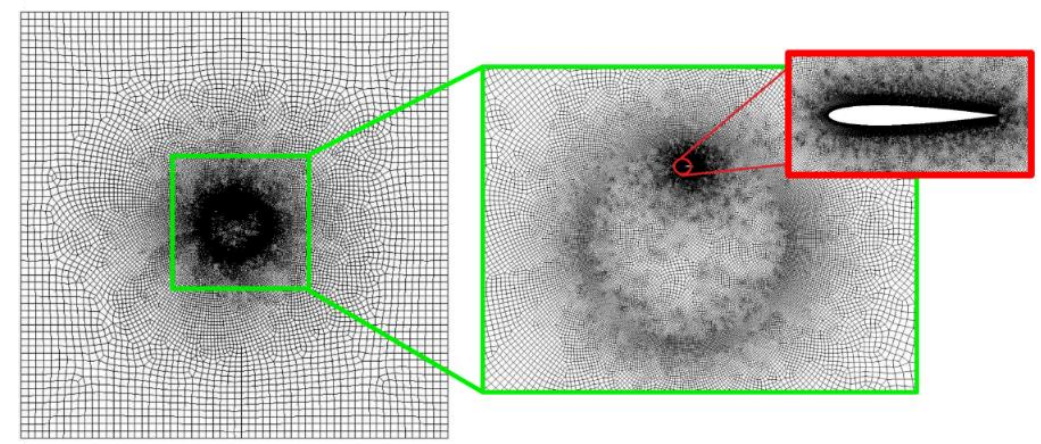

b)

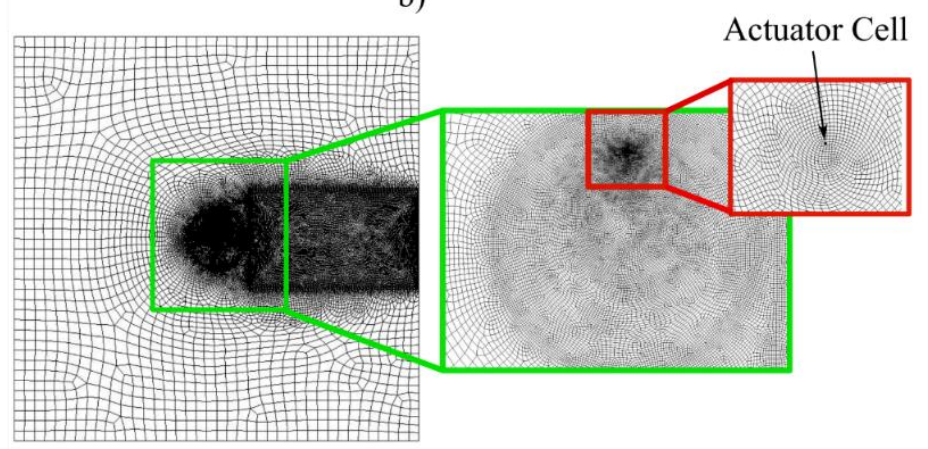

Fig. 3. Mesh for URANS simulations (a); Mech for ACM approach (b).

\section{Results and discussion}

Numerical results are given as aerodynamic blade loads and aerodynamic flow parameters. The non-dimensional tangential and normal force coefficients are expressed as:

$$
C F N, C F T=\frac{F_{N, T}}{0.5 \rho c V_{0}^{2}}
$$

where: $c$ is the chord length; $V_{0}$ is the velocity of the flow at the inlet; $\rho$ is the water density and $F_{N}$ and $F_{T}$ are aerodynamic blade load components (Figs $1 \mathrm{a}$ and $1 \mathrm{~b}$ ). Figure 4 presents computed and measured normal and tangential blade load components. Almost in the entire azimuth range, the numerical results agree with the experimental results. The largest discrepancies are in the case of the tangential force in the azimuth range from 100 to 180 degrees. They result from the local crossing of critical angles of attack. 
a)

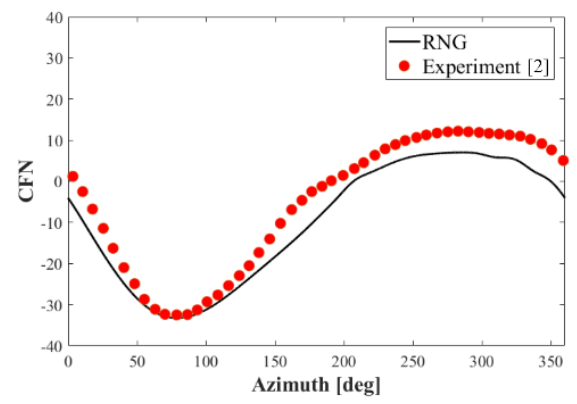

b)

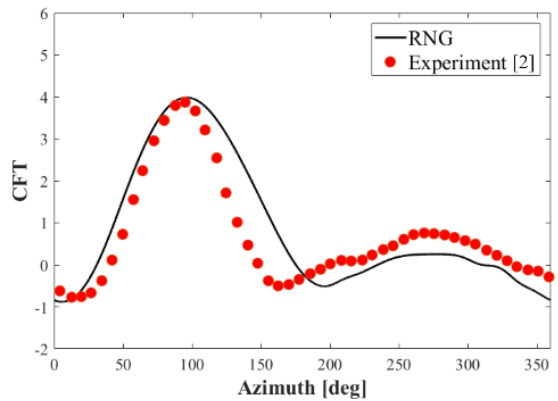

Fig. 4. Non-dimensional aerodynamic blade load coefficients as a function of azimuthal position: the normal component (a) and the tangential component (b).

The flow field at a distance of one rotor diameter behind the rotor was quantified by means of instantaneous velocity profiles for several azimuth positions. The numerical results were compared with the experimental results [2]. Velocity components are normalized by the velocity at the inlet $V_{0}\left(u=V_{x} / V_{0}\right)$. In the case of velocity profiles, two numerical approaches were used: the URANS model with the $k-\varepsilon$ turbulence model and the $\mathrm{ACM}$ approach. In the ACM method, the momentum sources were calculated based on experimental loads. As can be seen in the Fig. 5, the differences in velocity profiles are very small for all three sets of data.
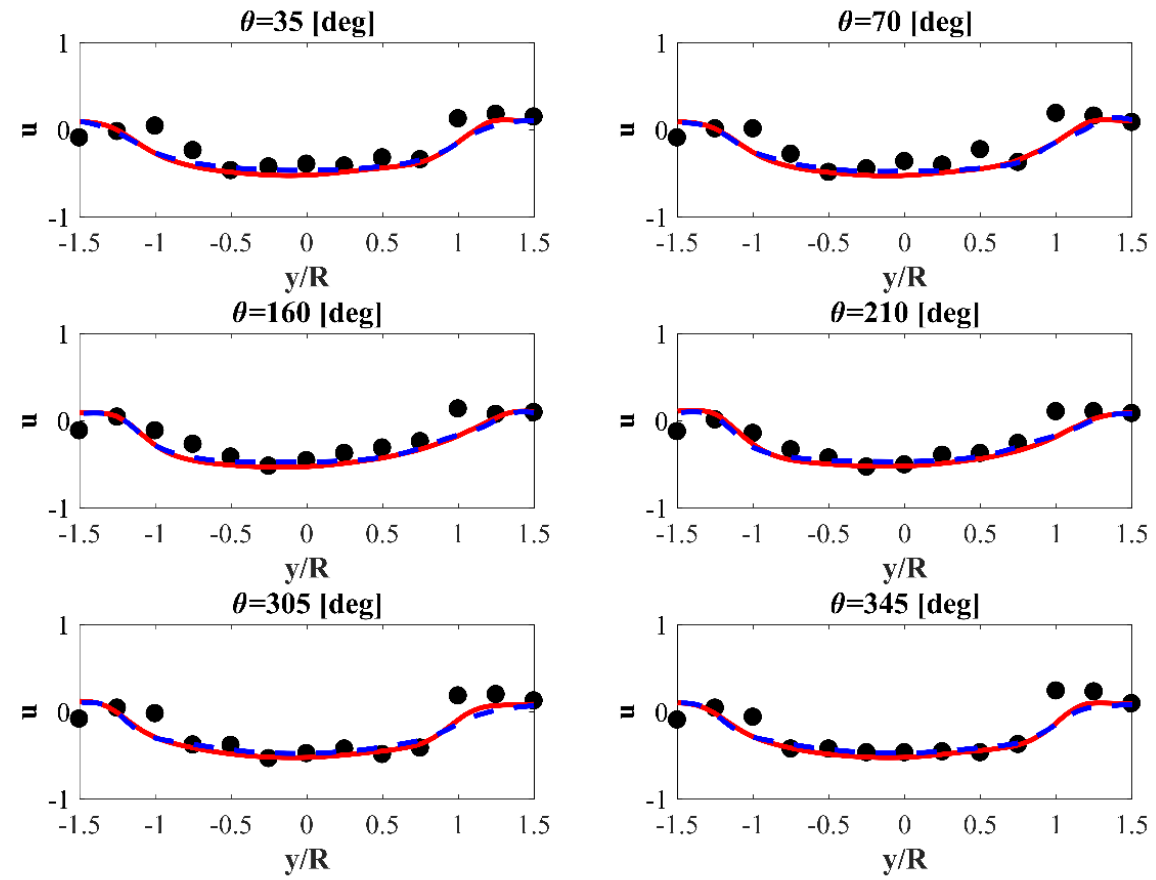

Fig. 5. Velocity profiles downstream behind the rotor: experiment [2] - black circles, $k-\varepsilon$ RNG - solid red line and ACM approach - blue dotted line.

Both numerical approaches were also validated by comparing streacklines. Streaklines represent the trajectory of solid particles or particles of other fluid injected into the fluid. In 
the case of Strickland's experimental studies [2], a dye was injected into the water, which was then photographed. The injector was located at one point under the water surface at the trailing edge of the blade. In the case of numerical methods, massless particles were used. These particles come out of the trailing edge of the blade profile or in the case of the ACM method from the third the smallest domain (from the active mesh cell). The visualizations given in Figure 6 show that the trajectories of particles are strongly curved. Despite large dissipation of particles within one-two diameters downstream behind the rotor, the motion images of particles obtained by two numerical approaches are agree with each other and with the experiment.

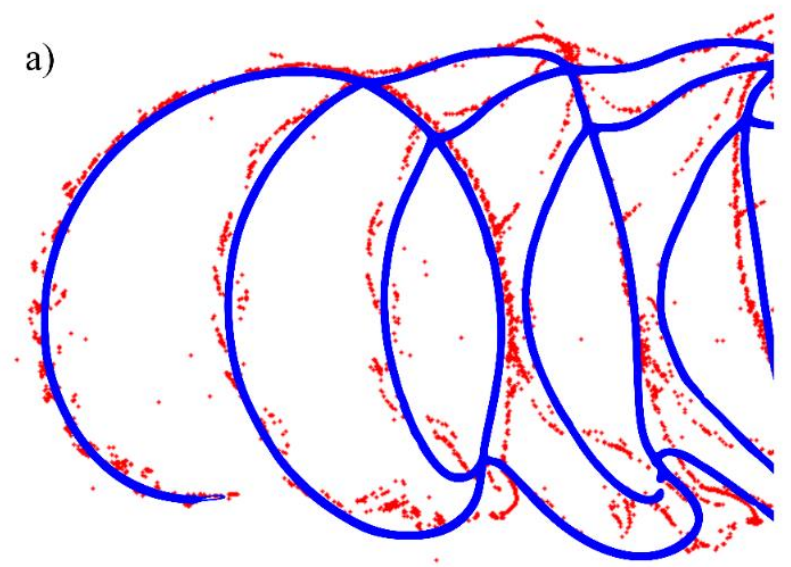

b)

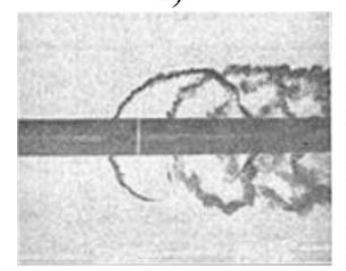

c)

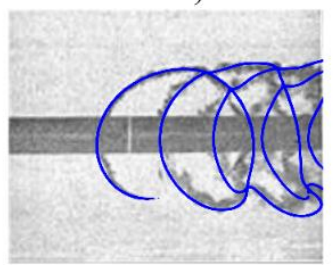

d)

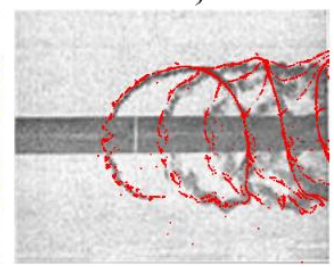

Fig. 6. Streaklines: the comparison between ACM (red colour) and URANS (blue colour) (a); experimental results [2] (b); the comparison between the experimental results [2] and URANS (blue colour) (c); the comparison between the experimental results [2] and ACM (red colour) (d).

Figure 7 shows contour maps of the component flow velocity $V_{x}$ for two numerical approaches. The velocity component $V_{x}$ is parallel to the undisturbed flow direction. The widths of the aerodynamic wakes on both numerical models are similar. The ACM model was coupled with the laminar model therefore some differences in velocity distributions are visible in Fig. 7. 


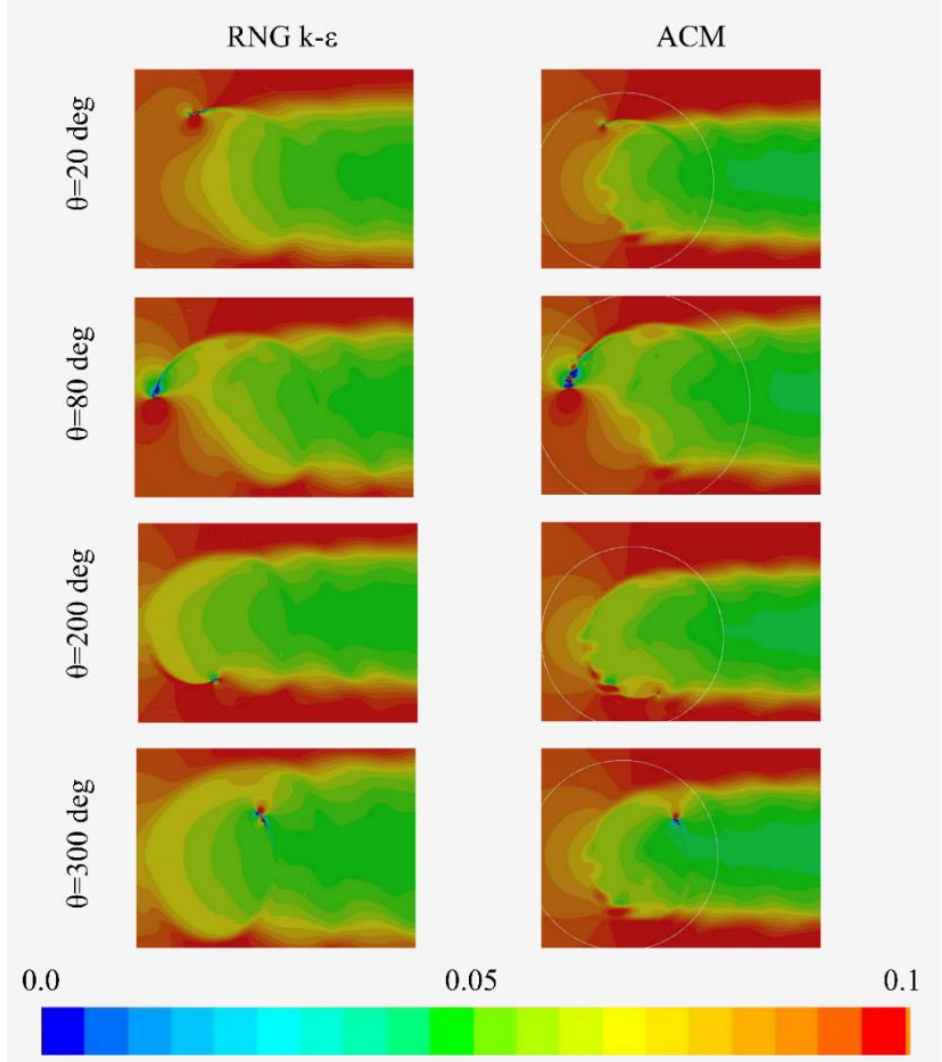

Fig. 7. Contour maps of velocity component $V_{x}[\mathrm{~m} / \mathrm{s}]$. Comparison of results for $k-\varepsilon$ RNG and ACM.

As assured in the Introduction, Darrieus's rotors are very effective devices. The efficiency of these devices can be measured using the so-called rotor power coefficient, $C_{P}$. It is commonly defined as the ratio of the energy produced by the turbine rotor divided by the total energy available in the wind at that velocity. Following the Paraschivoiu [3], the rotor power coefficient can be defined as the product of the averaged rotor torque coefficient, $\overline{C_{m}}$, and the tip speed ratio, TSR.

$$
C_{P}=\overline{C_{m}} \cdot T S R
$$

The tip speed ratio is defined as the ratio between the tangential velocity of the blade and the flow velocity. For a TSR value of 5 , the tangential velocity of the blade is 5 times greater than the undisturbed flow rate. The rotor power coefficient computed in the present study using the RNG k- $\varepsilon$ turbulence model is equal to 0.237 . The result presented by Strickland et al. [2] by using their vortex model code is 0.248 . The result obtained in these simulations is greater by $4.6 \%$ compared to the results of Strickland at al. [2].

\section{Conclusions}

The purpose of the paper was the analysis of the flow past the one-bladed vertical-axis wind turbine using two numerical approaches: URANS with the k- $\varepsilon$ RNG turbulence model and the home-made actuator cell model. 
The k- $\varepsilon$ RNG model gives satisfactory results of aerodynamic forces for the single-bladed rotor of the Darrieus wind turbine. The velocity profiles obtained using the URANS and ACM approaches are quite good compared to the experimental results. The ACM method presented by the authors is a development method. In this work it was proved that the flow field calculated with it gives results in accordance with the classical URANS approach and with the experiment. The high value of the rotor power coefficient for the one-bladed rotor indicates the high energy potential of the Darrieus wind turbine. The high rotational velocity makes it possible to use this type of wind turbine to produce electricity and transmit it to the grid.

The presented numerical computations were performed in the Interdisciplinary Centre for Mathematical and Computational Modelling of the Warsaw University, Grant No. GB73-5.

The author, Krzysztof Rogowski would like to thank the National Science Center for the possibility of a scientific trip to Denmark where the method and results described in this article were consulted.

\section{References}

1. E. A. D. Kumara, N. K. Hettiarachchi, K. G. R. M. Jayathilake, Int. J. Sci. Res. Inno. Techn. 4, 8, 56-67 (2017)

2. J. H. Strickland, B. T. Webster, T. Nguyen, Vortex Model of the Darrieus Turbine: An Analytical and Experimental Study (Tehnical raport SAN79-7058, 1979)

3. I. Paraschivoiu, Wind Turbine Design: With Emphasis on Darrieus Concept (Presses inter Polytechnique, 2002)

4. G. Bangga, G. Hutomo, R. Wiranegara, H. Sasongko, J. of Mech. Sc. and Techn. 31, 1, 261-267 (2017)

5. K. Rogowski, M. O. L. Hansen, P. Lichota, J. of Appl. Fluid Mech. 11, 4 (2018)

6. R. Rezaeiha, I. Kalkman, B. Blocken, Renewable Energy 107, 373-385 (2017)

7. J. H. Wiśniewski, Decreasing Wear of Large Vertical Axis Wind Turbines by Employing a Multi-Level Turbine (Concept Renewable and Alternative Energy: Concepts, Methodologies, Tools, and Applications, 2016)

8. P. Lichota, D. A. Noreña, A Priori Model Inclusion in the Multisine Maneuver Design $\left(17^{\text {th }}\right.$ International Carpathian Control Conference, IEEE, Tatrzańska Lomnica, Słowacja, 2016)

9. P. Lichota, K. Sibilski, P. Ohme, J. of Aircraft 54, 2 (2017)

10. U. S. Paulsen, H. A. Madsen, K. A. Kragh, P. H. Nielsen, I. Baran, J. Hattel, E. Ritchie, K. Leban, H. G. Svendsen, P. A. Berthelsen, Energy Procedia, 53 (2014)

11. D. Verelst, H. A. Madsen, M. Borg, U. S. Paulsen, H. G. Svendsen, P. A. Berthelsen, Energy Procedia, 80 (2015)

12. K. Rogowski, M. O. L. Hansen, R. Maroński, P. Lichota, J. Phys. Conf. Ser. 753, 022050 (2016)

13. K. Rogowski, J. Phys. Conf. Ser. 1101, 012028 (2018) 